\title{
La responsabilité sociale des entreprises : vers un nouveau consensus canadien
}

\author{
Par Marc-André Morency et Jeanne Simard ${ }^{1}$ \\ Université du Québec à Chicoutimi
}

En janvier 2002, la Commission sur la démocratie canadienne et la responsabilisation des entreprises ${ }^{2}$ déposait un rapport proposant une série de mesures visant à mieux responsabiliser les entreprises. Cette Commission examine comment nos entreprises pourraient faire preuve de responsabilité sociale envers les Canadiens et d'autres citoyens touchés par leurs opérations et ce, tout en demeurant compétitives dans une économie qui se mondialise. On sait que les corporations disposent d'un pouvoir et d'une influence de plus en plus considérables sur notre société et sur la scène mondiale. Mais en sont-elles redevables? En d'autres termes, rendent-elles compte de leurs actes et en assument-elles la responsabilité ?

\section{On sait que les corporations disposent d'un pouvoir et d'une influence de plus en plus considérables sur notre société et sur la scène mondiale. Mais en sont-elles redevables? En \\ d'autres termes, rendent-elles compte de leurs actes et en assument-elles la responsabilité?}

La pertinence d'un tel questionnement nous a été révélée ces derniers mois par l'affaire Enron-Anderson qui a démontré au monde entier que le décloisonnement et la déréglementation des services financiers et professionnels aux U.S.A. avaient ouvert la porte aux pires abus de confiance et à des conflits d'intérêts. L'ambition démesurée de certains dirigeants d'Enron a conduit l'entreprise à dépouiller, à l'aide de stratagèmes comptables analysés par la firme d'audit et de conseil Anderson, des milliers d'actionnaires américains et des salariés ${ }^{3}$. Cette affaire a profondément ébranlé la confiance des investisseurs et du grand public quant à la sécurité des investissements aux États-Unis et même ailleurs dans le monde. Cette insécurité est d'autant plus ressentie que certains dispositifs financiers conçus pour dissimuler les dettes d'Enron n'étaient pas nécessairement illégaux ${ }^{4}$.
La déréglementation de la finance aux États-Unis, dont l'ex-président du Federal Reserve Board (la Banque centrale privée américaine), Paul Volcker, avait été luimême un important artisan dans les années 1980, semble donc être allée beaucoup trop loin, car on attribue au déclin de la confiance la panne actuelle de l'économie mondiale ${ }^{5}$. Il en résulte que, présentement, aux ÉtatsUnis, au Canada, en Europe et certainement dans d'autres pays, on est forcé d'examiner si on n'a pas dépassé toutes les limites du raisonnable en matière de gouvernance d'entreprise et de sécurité des groupes touchés par les décisions des organisations. On a pu constater que les sanctions juridiques ou les réactions des investisseurs peuvent avoir des conséquences très graves pour la survie des entreprises et pour la santé de l'économie en général. On sait maintenant que Volcker, recruté afin de sauver Anderson, a dû constater l'échec de sa tentative de réorganisation de la firme, conduisant au démembrement du numéro cinq mondial dans ce domaine.

Au cours des derniers mois, on a assisté à un tel éclatement d'affaires touchant de grandes corporations américaines et étrangères (WorldCom, Tyco, Alcatel), que l'on observe un fléchissement marqué de l'activité boursière, effarouchée par les pratiques légales douteuses et l'encadrement éthique défectueux des entreprises ${ }^{6}$. La confiance s'effondre, les investisseurs se réfugient dans des valeurs dites sûres; les institutions, comme les consciences, opposent au libéralisme un cinglant démenti. On a évalué à 7 trillions de dollars US les pertes encourues dans la dégringolade boursière de l'été 2002. Ces évènements jettent une lumière toute particulière sur la défaillance des modes de gouvernance d'entreprise et, de façon plus globale, sur les processus en cours relativement à la responsabilité sociale des entreprises (RSE).

Mais avant même qu'éclatent ces scandales, plusieurs organisations nationales et internationales se penchaient 
sur la question de la responsabilité sociale de l'entreprise, cherchant à clarifier une notion encore floue sur le plan conceptuel et légal en raison notamment de la complexité des contextes sociaux touchés par l'activité des entreprises. Dans cet article, nous examinons le Rapport de la Commission sur la démocratie canadienne et la responsabilisation des entreprises qui propose des amendements aux lois canadiennes dont la portée semble restreindre indûment l'extension de la RSE.

\section{La Commission : origine, composition et processus de consultation}

Il est important de mentionner dès le départ que la Commission sur la démocratie canadienne et la responsabilisation des entreprises (ci-après appelée «la Commission») n'est pas un organisme gouvernemental. Elle a été mise sur pied par le Arthur Kroeger College of Public Affairs de l'Université Carleton. La Atkinson Charitable Foundation en était le commanditaire principal. La Commission est composée de cinq commissaires issus de différents milieux (affaires, syndicats et politique) dont deux coprésidents, Edward Broadbent (ex-chef du Nouveau parti démocratique du Canada) et Avie Bennett (chancelier de l'Université York).

Après s'être rendue aux quatre coins du pays aux fins de recueillir par voie de mémoires et d'audiences publiques les réactions de gens d'affaires, de groupes confessionnels, de représentants syndicaux, de chefs de gouvernement, d'investisseurs, d'universitaires et de citoyens concernés et après avoir réalisé un sondage auprès d'un peu plus de 2000 adultes, la Commission en est arrivée à dégager un consensus sous forme de 24 recommandations concrètes et pratiques en matière de responsabilité sociale des entreprises. Dans le rapport, disponible sur le site Web de la Commission depuis janvier 2002 (on y trouvera également les résultats du sondage, ainsi que le texte de différents mémoires), on peut lire des recommandations visant à amender principalement des textes de lois, à adopter des ententes, à rendre effective l'application de lois et de règlements en vigueur touchant les corporations, le marché des valeurs mobilières, le consommateur, la santé et la sécurité, les délits criminels et enfin l'environnement.

La Commission en est arrivée à dégager un consensus sous forme de 24 recommandations concrètes et pratiques en matière de responsabilité sociale des entreprises.
En effet, à la lecture du rapport de la Commission, on constate qu'à l'instar de nombreux intervenants, la Commission remet en cause le principe corporatif traditionnel de la «primauté des actionnaires» qui ne donne plus, selon elle, une représentation adéquate de la véritable responsabilité sociale des entreprises vis-à-vis les sociétés démocratiques actuelles. Les principales critiques formulées sont habituellement de deux ordres : la responsabilité des entreprises se réduit trop souvent à maximiser le rendement aux actionnaires au détriment des droits de la personne, des conditions de travail et de l'environnement d'une part, et, d'autre part, les pratiques abusives des dirigeants sont largement protégées par un système qui manque de transparence ou de rigueur dans l'application des lois et règlements.

Or, même si le profit est la première raison d'être d'une compagnie, celle-ci doit aussi être redevable auprès des groupes d'intérêt touchés par ses opérations, c'est-à-dire faire preuve de conscience sociale. Les nombreuses manifestations en faveur des droits de la personne et de l'environnement démontrent sans contredit que les compagnies sont appelées de plus en plus à se montrer bonnes citoyennes si elles ne veulent pas faire l'objet de boycotts et perdre leur réputation. La Commission souhaite voir combler le fossé qui se creuse entre le monde des entreprises et les autres partenaires de la société civile qui perdent confiance dans la gouvernance actuelle centrée sur le profit maximum, plutôt que sur la valeur maximum à long terme.

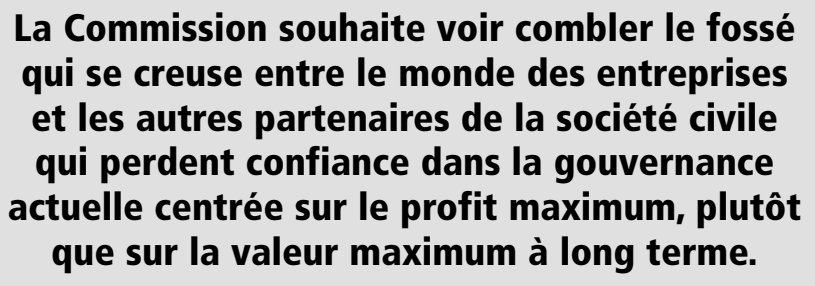

Afin de répondre à ces nouveaux critères et de remettre en cause la pertinence explicative du modèle traditionnel, d'autres solutions furent proposées. La Commission trouvait toutefois inopportun de retenir la position, opposée au modèle traditionnel, qui rejette la légitimité ou l'existence même des compagnies. La Commission a plutôt exploré la possibilité d'adopter des voies intermédiaires qui recherchent une conciliation, un juste milieu entre les positions extrêmes. La Commission rejette d'emblée un système où les gestionnaires désigneraient eux-mêmes les groupes d'intérêt auxquels ils seraient 
redevables, car il leur serait reconnu une trop grande marge de manœuvre dans la détermination de leur responsabilité. Par contre, elle privilégie une position où il serait accordé la même priorité aux groupes d'intérêt et aux objectifs des actionnaires et ce, à l'intérieur d'un cadre fixé par le législateur.

La Commission, selon toute évidence, cherchait à s'inscrire dans le processus de réforme de la législation fédérale dont la Loi canadienne sur les sociétés par actions (ci-après appelée LCSA) doit être en principe révisée à tous les cinq ans. Cette préoccupation pragmatique a marqué la nature des cadres d'analyse utilisés de même que la stratégie de consultation privilégiée qui s'inspire, croyons-nous, de la pratique du Livre vert de la Communauté européenne ${ }^{7}$ et des commissions itinérantes. La Commission a voulu démontrer l'existence d'un vaste consensus, facilitant d'autant la réforme de la législation.

Le rapport de la Commission revêt donc une importance certaine pour les quelque 150000 entreprises canadiennes visées par ces réflexions sur la RSE. Il est dommage, à cet égard, que ce rapport n'ait pas reçu toute l'attention qu'il méritait, à tout le moins au Québec, la presse ayant fait peu de cas de ce processus ${ }^{8}$. D'où l'intérêt pour les auteurs de cet article d'en souligner l'importance.

\section{L'effectivité des lois au Canada et les freins législatifs en matière de RSE}

Sur le plan national, la Commission semble généralement considérer comme adéquates les normes de RSE déjà prévues dans différents textes de loi du pays en matière de protection des consommateurs, d'environnement, de santé et sécurité au travail, etc. Par contre, elle s'interroge sur l'application de ces normes. Il semblerait que les entreprises délinquantes soient rarement sanctionnées, ce qui revient en quelque sorte à une abrogation ou à une déréglementation de facto. En conséquence, elle réclame des différents gouvernements du Canada qu'ils prennent les moyens nécessaires pour rendre effectifs les lois et les règlements en vigueur de notre pays (recommandation no 14).

Par contre, la Commission reconnaît qu'il n'est ni possible ni souhaitable d'implanter toute la RSE par la seule contrainte légale. À ce sujet, un témoin de la Commission, David Stewart-Patterson du Conseil canadien des chefs d'entreprises, soulignait ceci:
En tant que société, nous adoptons des lois et nous promulguons des règlements qui régissent les comportements. Nous agissons ainsi dans la mesure où il est possible de clairement distinguer le bien du mal... Cependant, les décisions des entreprises doivent tenir compte de multiples facteurs qui dépassent l'application pure et simple de la loi. Comme c'est le cas pour les discussions de nature éthique, il n'existe souvent pas de réponse claire. Par contre, les gestionnaires, les administrateurs et les actionnaires doivent prendre les meilleures décisions possibles en fonction des données dont ils disposent. ${ }^{9}$

\section{La Commission reconnaît qu'il n'est ni possible ni souhaitable d'implanter toute la RSE par la seule contrainte légale.}

Il s'avère en effet important, selon la Commission, que les entreprises ne soient pas privées de la flexibilité nécessaire à la gestion de leur responsabilité sociale dans les différents contextes qu'elles rencontrent. Toutefois, si les administrateurs d'une entreprise constituée en compagnie en vertu de la loi fédérale doivent proposer des normes qui vont au-delà du seuil minimal prévu dans les différentes lois (par exemple, activités philanthropiques, initiatives pour faciliter l'accès des personnes âgées ou à faible revenu à des services particuliers de l'entreprise), il s'avère important de clarifier leur responsabilité légale vis-à-vis la compagnie. En effet, l'obligation de fiduciaire que leur impose la LCSA peut être vue comme contraignant leurs démarches. Cette obligation leur impose de faire preuve, dans l'accomplissement de leurs devoirs, d'honnêteté, de bonne foi, et d'agir dans le meilleur intérêt de l'entreprise. Mais agir dans le meilleur intérêt de l'entreprise ne permet pas nécessairement aux administrateurs de consacrer des ressources à des initiatives de RSE qui vont au-delà des obligations légales déjà existantes 10 . En quelque sorte, les administrateurs seraient à la limite contraints d'agir avec pour seul objectif le bien de la compagnie, personne morale distincte, sans tenir compte des intérêts d'autres personnes, groupes ou entités. Il en résulte que ce seraient les actionnaires qui bénéficieraient de ce devoir d'honnêteté des administrateurs, car l'augmentation des profits de la compagnie tend généralement à augmenter le rendement des investissements. La Commission ne donne aucune recommandation concernant les programmes d'options (stocks options), pratique qui ouvre la porte aux pires conflits d'intérêt chez les fiduciaires-investisseurs. 
On notera ici que, selon la Commission, la LCSA oblige les entreprises à rechercher un rendement maximum. Cette loi serait en fait pratiquement incompatible avec la tendance marquée des entreprises ou des collectivités à définir la RSE, du fait que son implantation ne peut résulter que de motions émanant des actionnaires. Cette loi serait en fait très en retard sur la législation qui se développe aux U.S.A.

La logique mercantile du rendement ou ce que d'aucuns appellent «la primauté des actionnaires» fait l'objet depuis longtemps de nombreuses critiques. La Commission a pu d'ailleurs s'en rendre compte dans les commentaires qu'elle a recueillis à ce sujet durant ses travaux. C'est à ce problème d'intégration de la responsabilité sociale à la gestion de l'entreprise que la Commission s'est donc attaquée dans son rapport. Elle propose des amendements importants à la LCSA de façon à préciser la nature exacte des obligations fiduciaires afin que les administrateurs d'une compagnie soient désormais clairement autorisés à tenir compte de l'impact des activités de l'entreprise sur ses employés, ses clients, ses fournisseurs ainsi que sur les collectivités locales, bref sur ce que l'on désigne comme des stakeholders (recommandation no 12) ${ }^{11}$. Il est important toutefois de mentionner que la Commission est loin d'être persuadée que, dans les faits, un tel amendement sensibiliserait davantage les gestionnaires à la RSE. Une définition plus précise de l'obligation fiduciaire aura tout au plus, selon elle, le mérite de faire disparaître un frein légal à une éventuelle initiative de RSE en écartant la crainte de poursuites éventuelles. D'autres mesures seront nécessaires pour que la nouvelle culture d'entreprise prenne la place qui lui revient dans le monde contemporain (recommandation no 21).

La Commission se réjouit des modifications apportées en 2001 à la LCSA par le législateur fédéral. En effet, notre lecture du texte amendé de la loi montre qu'on y diminue les exigences quant à l'admissibilité des propositions d'actionnaires en matière de RSE. Désormais, les véritables propriétaires d'au moins $1 \%$ des actions avec droit de vote (ou dont la valeur au marché est d'au moins 2000 \$), qu'ils soient ou non inscrits aux registres de la société, auront plus de facilité à faire ajouter à l'ordre du jour des assemblées annuelles d'actionnaires, des propositions qui ont pour principal objet de servir des fins générales d'ordre économique, politique, religieux, social ou autre. Avant l'amendement de la LCSA, une société pouvait facilement faire rejeter une proposition n'ayant pas vraiment de rapport avec ses activités com- merciales ou ses affaires internes. Il s'agit maintenant de vérifier si, dans les faits, les actionnaires seront capables de s'exprimer au sujet de la RSE aux réunions d'actionnaires (recommandation no 11). De plus, les modifications à la LCSA visent à soutenir la participation des actionnaires à la gouvernance d'entreprise en facilitant leurs communications et leur regroupement. En effet, les règles concernant la sollicitation de procurations ont été assouplies de façon à exclure de cette définition l'annonce publique, par un actionnaire, de ses intentions de vote et de ce qui les motive. Par conséquent, il est désormais permis aux actionnaires d'exprimer leurs opinions, «notamment dans le cadre de discussions informelles ou de lettres personnelles critiquant la direction ${ }^{12}$. Ils ne seront plus passibles d'une amende ou d'une peine de prison.

La Commission s'est penchée sur certains aspects relatifs à la gouvernance d'entreprise et à la formation. Elle recommande la création de comités consultatifs de la RSE relevant des conseils d'administration, la nomination d'ombudsmans de la RSE et l'amélioration de la formation des administrateurs dans les écoles de haut savoir (recommandations no 21 et 22).

\section{La Commission s'est penchée sur certains aspects relatifs à la gouvernance d'entreprise et à la formation. Elle recommande la création de comités consultatifs de la RSE relevant des conseils d'administration, la nomination d'ombudsmans de la RSE et I'amélioration de la formation des admi- nistrateurs dans les écoles de haut savoir.}

Enfin, la Commission croit que le gouvernement fédéral a un rôle important à jouer afin d'encourager les entreprises à agir de façon responsable. Au-delà de son pouvoir réglementaire, le gouvernement devrait selon elle obliger les entreprises de 100 employés ou plus à certifier leur respect de toutes les normes énoncées dans leurs lignes directrices de RSE avant tout octroi de sa part d'un contrat de vente de biens ou de services de plus de $200000 \$$. Cette même exigence devrait s'appliquer à tout octroi aux entreprises de subventions ou de certains avantages (recommandation no 15).

\section{L'entreprise canadienne à l'étranger}

Une des grandes préoccupations de la Commission a trait au comportement des entreprises canadiennes qui 
opèrent de plus en plus fréquemment dans des pays dépourvus de lois sociales analogues à celles de notre pays. Qu'advient-il dans ces pays du respect des droits de la personne? Des droits des travailleurs? De la protection de l'environnement et du consommateur?

La Commission ne croit pas que les lois du marché et l'impulsion de la société civile, même si elles jouent un rôle important, suffisent à élever la RSE au rang de pratique généralisée; elles n'assurent pas non plus un seuil commun au jeu de la concurrence entre acteurs de la vie économique. Notamment, les firmes qui ne produisent pas directement pour le consommateur demeurent largement inconnues et peu sensibles au boycott de leurs produits, c'est-à-dire peu sensibles à la société civile et à l'opinion. Les codes volontaires des entreprises ne représentent pas à ses yeux un substitut aux lois et aux règles contraignantes d'un pays. Par conséquent, la Commission favorise le recours au droit en proposant l'ajout aux ententes commerciales internationales de clauses qui comporteraient des normes sociales minimales et coercitives afin d'encadrer les activités des entreprises canadiennes à l'étranger (recommandation no 17).

Contrairement à certains organismes comme le Conference Board of Canada, le Comité inter-Églises ou le Canadian Business for Social Responsability, la Commission n'essaie pas de définir avec précision un ensemble de normes de RSE qui permettraient d'évaluer les entreprises. Elle propose tout au plus un cadre délimitant les «règles du jeu» à l'intérieur duquel les entreprises pourront exercer leurs activités à l'étranger. Ces règles du jeu incluraient les droits fondamentaux définis par la Déclaration universelle des droits de l'homme et les lois internationales en matière de droits de la personne, les normes fondamentales du travail proposées par l'OIT, par l'ONU (le Contrat mondial) et par l'OCDE, relativement à la libre association, à l'interdiction d'exploiter la main-d'œuvre enfantine ou le travail forcé, et relativement à la discrimination dans le travail ${ }^{13}$. La Commission recommande que les normes canadiennes en matière de protection du consommateur et de l'environnement soient respectées par les entreprises oeuvrant dans des pays qui ont des normes moins rigoureuses. La question de l'extraterritorialité relève du politique et non du droit, selon la Commission ${ }^{14}$.

La Commission va jusqu'à inciter le gouvernement fédéral à militer en faveur de l'ajout d'ici trois ans de ces normes minimales aux règles commerciales de l'Organi- sation mondiale du commerce et aussi de faire porter ses actions sur l'ensemble des institutions mondiales susceptibles de réguler la mondialisation. Cette régulation serait alors conforme aux valeurs fondamentales des Canadiens, leur appui à la mondialisation en dépendant (recommandation no 18). Si aucun progrès notable n'est accompli passé ce délai, on propose au gouvernement canadien d'agir unilatéralement et d'amender les textes législatifs de façon à obliger même les compagnies étrangères, dès lors qu'elles oeuvrent en sol canadien, à respecter les normes minimales en matière de droits humains et ce, sous peine de sanction (recommandation no 20).

La portée des recommandations de la Commission peut être schématisée comme suit:

\section{Cadre minimal de RSE au plan international pour les entreprises canadiennes}

\begin{tabular}{|c|c|c|c|}
\hline \multirow{4}{*}{$\begin{array}{c}\text { Lois } \\
\text { canadiennes }\end{array}$} & Lois ca & ennes & \multirow{4}{*}{$\begin{array}{c}\text { Déclaration } \\
\text { universelle } \\
\text { des droits } \\
\text { de l'homme }\end{array}$} \\
\hline & Envirc & ment & \\
\hline & $\begin{array}{l}\text { Protection } d u \\
\text { consommateur }\end{array}$ & $\begin{array}{l}\text { Droits de } \\
\text { la personne }\end{array}$ & \\
\hline & \multicolumn{2}{|c|}{ Protection du travailleur } & \\
\hline $\begin{array}{r}\mathrm{Nc} \\
\mathrm{F}\end{array}$ & $\begin{array}{l}\text { Règle } \\
\text { mes prévues au } \\
\text { incipes directeur }\end{array}$ & $\begin{array}{l}\text { OIT } \\
\text { rat mondial }(\mathrm{O} \\
\text { ncés par l'OC }\end{array}$ & \\
\hline
\end{tabular}

\section{La transparence et l'imputabilité de l'entreprise}

Que ce soit sur le plan national ou international, la Commission considère la transparence des entreprises comme une condition essentielle à la promotion de la RSE. En effet, tout investisseur a besoin d'information pour prendre une décision éclairée au sujet des entreprises qui l'intéressent. Or, présentement, aucune disposition législative n'oblige les entreprises à divulguer des données sur leurs pratiques de RSE; la LCSA impose tout au plus aux entreprises de dévoiler à leurs actionnaires des renseignements concernant leur gestion financière. Les différentes lois sur les valeurs mobilières au Canada, quant à elles, les obligent à mettre au jour des renseignements de nature matérielle, seulement s'ils influencent de façon significative le prix courant ou la valeur marchande de la valeur mobilière. Par conséquent, l'obligation de divulguer des renseignements concernant la RSE devient 
pressante seulement si ces derniers ont une incidence défavorable sur le bénéfice net de l'entreprise.

Devant l'insuffisance des pratiques actuelles de communication (la transparence volontaire ne suffit pas), la Commission prône un renforcement de la législation canadienne en cette matière. Elle souhaite d'une part que s'implante la divulgation obligatoire par les entreprises de leurs politiques de RSE, qu'elles montrent la place qu'elles accordent à la responsabilité sociale, les règles qu'elles suivent et la nature de leur code de déontologie et, d'autre part, qu'elles fournissent dans leur rapport annuel un bilan social découlant de la mise en œuvre de leur politique et lignes directrices de RSE (recommandation no 8) ${ }^{15}$.

Dans le bilan social, on retrouverait des indicateurs de performance sociale et environnementale ainsi qu'une liste de toutes les offenses criminelles graves ou les infractions importantes à un règlement dont une entreprise a été reconnue coupable (recommandations no 5 et 6). Pour les compagnies privées, on limite toutefois cette obligation en fonction d'une taille qui n'a pas été définie par la Commission ${ }^{16}$.

Pendant les audiences, les membres de la Commission ont réalisé que la compétitivité internationale du Canada constituait la principale source de préoccupation en matière d'application de la RSE. Une amélioration de la transparence et de la réputation de nos entreprises les priveraitelles d'un avantage concurrentiel dans une économie mondialisée? La Commission répond par la négative à cette question, d'une part parce qu'elle limite l'application des exigences supplémentaires qu'elle propose en matière de RSE aux grandes corporations et aux entreprises contrôlées par elles, tout en dispensant, d'autre part, les petites organisations du fardeau que représente le bilan social (recommandation no 2) ${ }^{17}$.

Enfin, la Commission conclut son rapport en rappelant que le Canada accusera un sérieux retard en matière de RSE vis-à-vis d'autres pays s'il refuse d'adopter ses recommandations. En effet, le Royaume-Uni possède déjà son ministre de la RSE; de nombreux États américains ont élargi les pouvoirs des conseils d'administration des entreprises pour leur permettre de tenir compte d'un plus grand nombre de groupes d'intérêt et enfin l'Union européenne publiait à l'été 2001 un livre vert sur la RSE. Or, jusqu'à présent, à notre connaissance les recommandations de la Commission n'ont pas encore fait l'objet d'une étude parlementaire. On peut prévoir cependant qu'elles seront considérées lors de la prochaine révision quinquennale de la LCSA.
En résumé, la Commission recommande les mesures suivantes:

- éliminer les obstacles législatifs au développement de la RSE;

- préciser la nature exacte des obligations fiduciaires des administrateurs des compagnies et améliorer la gouvernance d'entreprise (comité de RSE, ombudsman, formation améliorée des futurs administrateurs en matière d'éthique et de RSE);

- supprimer les contributions politiques aux partis, aux candidats, aussi bien de la part des syndicats que des entreprises;

- obliger la publication de bilans sociaux audités;

- adopter des mesures incitatives à la RSE pour toute grande compagnie;

- fixer un cadre de RSE minimal pour les entreprises oeuvrant à l'extérieur du Canada;

- voir à l'application effective des lois et règlements déjà existants au Canada.

\section{Nos constats}

Nous constatons que la Commission s'est fixée pour principal objectif d'obtenir un amendement majeur à la LCSA afin de sortir l'administration des compagnies de l'emprise exclusive de l'objectif de rendement maximal pour l'investisseur. On notera cependant que la Commission n'a pas abordé les problèmes de conflits d'intérêt engendrés par les programmes d'options et l'usage planifié des lois sur la faillite, problèmes qui touchent principalement la gouvernance d'entreprise. Or face aux difficultés majeures ayant affecté l'économie durant ces derniers mois, de nombreuses voix se sont élevées pour que l'on agisse sérieusement pour contrer l'absence de contre-pouvoirs, l'abus de confiance et les malversations des dirigeants. À cet effet, Dominic D'Alessandro, président de la Financière Manuvie, formule des recommandations claires: «Poursuites et même emprisonnement pour les coupables d'actes illégaux, séparation des postes de président du conseil et de chef de la direction, et sérieux ménage dans les programmes d'options qu'il qualifie de grotesques» ${ }^{18}$. Le président de la Réserve fédérale de New York vient de se joindre à ce concert critique, relativement à la démesure des rémunérations versées aux dirigeants d'entreprise. William McDonough a estimé à 400 pour 1 le ratio des rémunérations versées aux cadres par rapport au salaire des ouvriers de production.

À commencer par les entreprises les plus solides, les directeurs généraux et leurs conseils d'administration 
devraient tout simplement arriver à la conclusion que les rémunérations des dirigeants sont excessives et les ajuster à des niveaux plus raisonnables et plus justifiables [...].

Ne devrait-il pas y avoir des limitations économiques et morales aux fossés créés par le système de rémunération dominé par le marché ? 19

Il est clair que la Commission ne pouvait répondre en 2001 aux problèmes que les affaires Enron-Anderson, etc. allaient nous permettre de découvrir durant l'année 2002. Et en ce sens, un cadre de référence plus large aurait permis de faire des propositions touchant les rapports entre l'entreprise et ses contextes sociaux, les conflits d'intérêt entre actionnaires et actionnaires-administrateurs. Bref, la Commission aurait mieux traité les problèmes de gouvernance d'entreprise qui empêchent la RSE d'occuper la place qui lui revient. Les propos judicieux du professeur Jean-Marie Chevalier ${ }^{20}$, concernant l'affaire Enron-Anderson, mérite qu'on en cite un long extrait:

[...] ce qui est sûr, c'est que le mode de gouvernement d'entreprise mis en place par Enron était intrinsèquement mauvais depuis le début.

Le gouvernement d'entreprise conditionne la marche de l'entreprise à court, moyen et long termes; il est aussi un moyen important d'allocation de la valeur créée par l'entreprise. Cette valeur doit être partagée entre les différents agents qui, directement ou indirectement, participent à l'entreprise et que l'on appelle les stakeholders: actionnaires, managers, salariés, fournisseurs et banquiers, consommateurs. C'est sur cette répartition de la valeur que l'entreprise génère sa réputation et la confiance qui lui est faite. Sur ce plan, les dirigeants d'Enron ont menti, trompé, trahi, pour reprendre les termes mêmes d'une couverture de Fortune Magazine. Ils ont dissimulé les comptes, ils ont volé l'entreprise, ils ont ruiné une partie de leurs salariés en leur imposant des plans de retraite investis en actions d'Enron.

[...] Le gouvernement d'entreprise est un enjeu majeur pour le fonctionnement efficace de l'économie de marché. Il doit assumer sa responsabilité à court, moyen et long termes face aux différents groupes concernés.

Faute de trouver un cadre pleinement adéquat en matière de gouvernance ne risquerait-on pas de voir la RSE uti- lisée comme simple outil de propagande ou, pire, comme une autre avenue permettant l'abus de biens sociaux ?

Cependant, force est de constater que la Commission n'a pas distingué toujours avec précision ce qui relève de l'éthique, de la déontologie et du droit ${ }^{21}$. Elle erre quelque peu en logeant, dans son tableau appelé «Un tableau de normes minimales», sous la rubrique «Éthique», des obligations juridiques, déontologiques ou morales en matière de corruption et d'intégrité des dirigeants. Cette confusion est malheureusement encore très fréquente et soutient des discours contradictoires sinon trompeurs.

\section{Force est de constater que} la Commission n'a pas distingué toujours avec précision ce qui relève de l'éthique, de la déontologie et du droit.

Enfin, les contextes où opèrent les entreprises ne sont pas autrement distingués. Il en résulte que l'implantation de la RSE ne peut guère aller jusqu'à permettre l'incorporation d'un cadre d'éthique et de ses moyens d'analyse, d'une façon aussi diversifiée que le commandent les contextes sociaux du monde actuel. La Commission aurait pu donner quelques exemples mettant en évidence l'action responsable d'entreprises dans une sélection de contextes sociaux. La Commission européenne en a d'ailleurs fait un de ses sous-objectifs importants.

Ces limitations du rapport ne doivent cependant pas faire oublier le consensus très important qu'a reconnu ou défini la Commission. Ce consensus présente à notre horizon une chance véritable d'inscrire dans la législation canadienne des amendements fort significatifs en matière de RSE. S'ils étaient mis en place, ces amendements permettraient au Canada de ne pas être trop en retard et même, comme le souhaite la Commission, de «combler le fossé» par rapport à ce qui peut être observé en Europe comme consensus émergent.

\section{Notes et références}

Marc-André Morency est professeur de sociologie au Département des sciences humaines et directeur du Module des sciences humaines, à l'Université du Québec à Chicoutimi. Jeanne Simard est avocate et professeure de droit au Département des sciences économiques et administratives, à l'Université du Québec à Chicoutimi; les auteurs tiennent à remercier Me Denis Bourque pour ses judicieuses remarques et M. Roger de la Sablonnière pour l'aide apportée en matière documentaire. 
2 Commission sur la démocratie canadienne et la responsabilisation des entreprises (2002). Rapport final: Une nouvelle équation; Les profits et les responsabilités des entreprises à l'aube du 21e siècle. Janvier. Ce document peut être consulté à l'adresse suivante: http://www.corporateaccountability.ca.

3 Les cadres supérieurs auraient empoché quelques 740 millions US, alors même qu'ils encourageaient leurs employés et leurs actionnaires à maintenir leur confiance et à garder leurs actions; eux-mêmes en profitaient pour liquider leurs options en toute hâte, la valeur des actions ayant commencé à décliner. Les autorités songeraient à en forcer la restitution.

4 Dans un éditorial du Devoir, on mentionnait ironiquement qu'à la suite de la dégringolade financière d'Enron, des biens de cette firme ont été mis aux enchères sur Internet, dont un «code d'éthique» (sic) au prix de 162\$!; Jean-Claude LECLERC (2002). «Enron ou le triomphe des conflits d'intérêts», Le Devoir, le 28 janvier; voir aussi sur l'affaire Enron: Michel DRANCOURT (2002). "Capitalisme: vers le grand nettoyage», Futuribles. Vol. 276, p. 71.

5 Voir William GREIDER (1987). Secrets of the Temple, How the Federal Reserve Runs the Country. New York, Simon and Schuster, $798 \mathrm{p}$.

6 Agence France-Presse Reuters, «Les marchés s'enfoncent: la crise de confiance des investisseurs dans les sociétés américaines a pesé sur la Bourse», Le Devoir, le 4 juin 2002; voir également dans RFI actualité, du 17 juin 2002 une analyse semblable de Francine QUENTIN : «Le G7 incite les marchés à se ressaisir». Dans le Devoir du 11 septembre 2002, François Dupuis, chef économiste-adjoint du Mouvement Desjardins, affirme que les affaires qui se sont déclarées en 2002, ont affecté l'économie d'une manière plus importante que les attentats terroristes du 11 septembre 2001; voir Éric DESROSIERS, «Enron et Worldcom ont fait plus de mal que le 11 septembre», p. B-4.

7 Commission des Communautés européennes (2001). Livre vert: Promouvoir un cadre européen pour la responsabilité sociale des entreprises, juin. Ce document peut être consulté à l'adresse suivante: http://europa.eu.int/comm/employment social/ soc-dial/csr/greenpaper fr.pdf.

8 Une commission parlementaire du Québec a néanmoins amorcé depuis mai 2002 une importante étude visant la réforme du droit québécois en matière de RSE. Ces travaux sont d'ailleurs maintenant accessibles sur le site suivant: http://www.assnat.qc.ca/fra/publications/rapports/concfp1.htm.

9 Id, p. 5
10 Notons au passage que les entreprises contribuent volontiers aux partis politiques, une pratique assez répandue et qui est contestée tant par la Commission que par une majorité de Canadiens.

11 Les recommandations de la Commission s'appliquent aussi aux caisses de retraite. On désire donc un amendement à la Loi sur les pensions afin de permettre aux administrateurs de fonds de pension de tenir compte de considérations non financières dans le cadre d'une décision d'investissement (recommandation no 13).

12 Margaret SMITH (2000). La loi canadienne sur les sociétés par actions; communications relatives aux actionnaires. Parliamentary Research Branch, Vol. 99, No. 33, 18 janvier.

13 En ce qui a trait aux relations de travail, toujours selon la Commission, certaines normes canadiennes ne peuvent s'appliquer intégralement et sans effets discriminants aux activités commerciales à l'étranger. Les entreprises risqueraient de perdre alors un avantage compétitif légitime.

14 La SEC américaine demande désormais aux compagnies étrangères oeuvrant aux États-Unis de certifier leurs bilans, sans que cela ne soulève vraiment de problème.

15 Dans le même esprit, on réclame que la Loi sur l'étiquetage des textiles soit amendée pour permettre aux consommateurs de savoir non seulement dans quel pays, mais également dans quelle usine ont été fabriqués les produits qu'ils achètent (recommandation $\mathrm{n}^{\circ} 7$ ).

16 Il serait intéressant de voir le Canada prendre le relais de la Thaïlande qui a récemment, selon Stiglitz, inséré dans sa constitution le droit à l'information (the right to know) pour ses citoyens, droit qui paraît essentiel au fonctionnement des marchés tout autant que de leur démocratie.

17 Par ailleurs, pour permettre une plus grande transparence en matière de RSE, les employés qui signalent une infraction ou un crime commis par leur employeur devraient être protégés contre tout congédiement, suspension, harcèlement ou mise sur une liste noire. On remarque ici que la Commission veut protéger l'employé qui dénonce un acte criminel, mais pas celui qui mettrait en doute une décision de nature éthique (recommandation $\mathrm{n}^{\circ} 10$ ).

18 Diane BÉRARD (2002). «Cupidité inc.», Commerce, Vol. 103, No. 7, Août, p. 32.

19 REUTERS (2002). «McDonough critique la gourmandise des dirigeants », Le Devoir, 12 septembre.

20 Directeur du Centre de géopolitique de l'énergie et des matières premières à l'université Paris-Dauphine, directeur du European Gas and Power au Cambridge Energy Reserch Associates.

21 À ce propos, voir Jeanne SIMARD et Marc-André MORENCY (2002). «Droit, déontologie et éthique: distinctions et applications en gestion», Organisations et territoires. Vol. 11, No. 2, pp. 13-18. 